\title{
ARTICLE OPEN \\ Facile measurement of single-crystal elastic constants from polycrystalline samples
}

\author{
Xinpeng $\mathrm{Du}^{1}$ and Ji-Cheng Zhao (iD)
}

Elastic constants are among the most fundamental properties of materials. Simulations of microstructural evolution and constitutive/micro-mechanistic modeling of materials properties require elastic constants that are predominately measured from single crystals that are labor intensive to grow. A facile technique is developed to measure elastic constants from polycrystalline samples. The technique is based upon measurements of the surface acoustic wave velocities with the help of a polydimethylsiloxane film grating that is placed on a polished surface of a polycrystalline sample to confine surface acoustic waves that are induced by a femtosecond laser and measured using pump-probe time-domain thermoreflectance. Electron backscatter diffraction is employed to measure the crystallographic orientation along which the surface acoustic wave propagates in each grain (perpendicular to the polydimethylsiloxane grating). Such measurements are performed on several grains. A robust mathematical solution was developed to compute the surface acoustic wave velocity along any crystallographic orientation of any crystal structure with given elastic constants and density. By inputting various starting values of elastic constants to compute the surface acoustic wave velocities to match experimental measurements in several distinct crystallographic orientations using an optimization algorithm, accurate elastic constant values have been obtained from seven polycrystalline metal samples to be within $6.8 \%$ of single-crystal measurements. This new technique can help change the current scenario that experimentally measured elastic constants are available for only about $1 \%$ of the estimated 160,000 distinct solid compounds, not to mention the significant need for elastic constants of various solid solution compositions that are the base of structural materials.

npj Computational Materials (2017)3:17; doi:10.1038/s41524-017-0019-x

\section{INTRODUCTION}

Single-crystal elastic constants are essential data not only for constitutive and micro-mechanistic modeling of various properties (especially mechanical properties including plastic deformation and fracture), ${ }^{1,2}$ but also for simulations of microstructure evolution during phase transformations and materials processing. ${ }^{3,4}$ Moreover, since single crystals are directly used in many applications from microelectronic devices to jet engine turbine blades, anisotropic elastic properties are essential for the design of those devices and components.

Even though significant progress has been made in computing the elastic constants using density functional theory, ${ }^{5}$ accurate experimental measurements are still essential until the computed results are fully validated for accuracy.

The elastic constants are predominately obtained from measurements on relatively large single crystal samples. Several experimental techniques have been developed to facilitate the measurement of elastic constants such as the resonant acoustic spectroscopy (RUS), ${ }^{6,7}$ and the Brillouin scattering. ${ }^{8,} 9$ These methods, albeit simpler in measurements, still require single crystals that are usually labor-intensive and time-consuming to grow. The RUS technique has been applied to measure elastic constants from micro-single-crystal samples of several hundred microns in dimensions and it has the advantage of being able to perform temperature-dependent measurements when the apparatus is enclosed within an inert environment.
Many compounds cannot even be grown into single crystals. As a result, experimental values of elastic constants of about $99 \%$ of the estimated 160,000 distinct solid compounds are unavailable (Rodgers, J.A., Private Communication, December 2014), ${ }^{10}$ not to mention the vast amounts of solid solution compositions that are the base of structural alloys. In the absence of elastic constants for solid solutions, the anisotropic properties of the pure elements are often used as model inputs for the solid solutions, which can lead to significant error in the simulated/computed properties.

It is highly desirable to extract single-crystal tensor data from polycrystalline samples, and it is even more desirable to perform measurements on one or two polished surfaces of a polycrystalline sample. One can imagine the usefulness of such measurements on multi-phase samples where one can obtain the elastic constants for different phases without making monolithic single-phase single crystals.

A so-called acoustic spectro-microscopy (ASM) was developed in the late 1990 s to measure elastic constants from polycrystalline $\mathrm{Ni}$ with $\sim 1 \mathrm{~mm}$ diameter grains. ${ }^{11,}{ }^{12}$ The method requires an extremely high accuracy of eucentricity during the rotational ASM measurements as well as painstaking (within $0.5 \mu \mathrm{m}$ ) alignment of the optical microscopy unit with the ASM unit when the sample is switched between them. The ASM measurements also rely on the use of a transducer liquid such as water, adding complexity and constraint to the type of samples to be measured. For these reasons, the method was not widely used.

${ }^{1}$ Department of Materials Science and Engineering, The Ohio State University, Columbus, OH, USA

Correspondence: Ji-Cheng Zhao (zhao.199@osu.edu)

Received: 19 November 2016 Revised: 18 March 2017 Accepted: 23 March 2017

Published online: 24 April 2017 
Very recently a laser pump-probe measurement was developed to extract elastic constants from a coarse-grained polycrystalline stainless steel sample by measuring the surface acoustic waves (SAWs). ${ }^{13}$ The method is only applicable to cubic crystal structures with the assumption of a random distribution of the crystal orientations and requires measurements on about a thousand grains; thus it can only be applicable to samples satisfying these stringent conditions. Other methods are developed to extract elastic constants from highly textured samples only. ${ }^{14,15}$

Nanoidentation modulus measurement coupled with orientation measurement using electron backscatter diffraction (EBSD) have recently been applied to obtain orientation-dependent Young's modulus, but the method has yet demonstrated the capability of evaluating the full elastic constant values. ${ }^{16}$ Atomic force microscopy (AFM)-based methods have extremely high spatial resolutions and can quickly produce qualitative modulus maps, ${ }^{17,} 18$ but extraction of accurate elastic constants from the AFM modulus maps is still far from a reality, especially for hard materials such as metals, inorganic semiconductors, and ceramics.

The facile technique to be established in the current study is built upon two prior studies. A robust mathematical solution was first developed to compute the SAW velocity along any crystallographic orientation of any crystal structure type with given elastic constants and density. ${ }^{19}$ Such a solution with inclusion of the thermal expansion effect induced by localized heating of a Gaussian-shaped laser beam is essential to accurately correlate the SAW velocities to the elastic constant values. The solution includes full computation of all the bulk waves, including the longitudinal (L) mode, the fast transverse (FT) mode, and the slow transverse (ST) mode, as well as SAWs including both the Rayleigh wave (RW) and the pseudo surface acoustic wave (PSAW).

Another development is an easy-to-implement and accurate measurement of the SAW velocity with a spatial resolution of $10-15 \mu \mathrm{m} .^{20}$ A polydimethylsiloxane (PDMS) film with parallel line grating is precast from a commercially available Si grating mold with $700 \mathrm{~nm}$ periodicity and $50 \%$ duty cycle. The PDMS film grating is placed on a sample with the grating lines in contact with the polished surface. A femtosecond laser with a beam diameter of $\sim 5 \mu \mathrm{m}$ is employed to generate the SAWs by passing through the transparent PDMS film grating. The SAW velocity perpendicular to the PDMS grating direction is measured from the frequency of the time-domain thermoreflectance (TDTR) signal of the probe beam that was split from the same laser. ${ }^{20}$ The advantages of the PDMS grating-assisted measurements include that: (1) the dispersion due to the broadband signal on the anisotropic surface is suppressed; (2) only the SAW perpendicular to the PDMS grating lines survives; and (3) the SAW velocity can be accurately measured as demonstrated along several known high-symmetry crystallographic orientations of single crystal samples. $^{20}$ The excellent agreement between the experimental measurements and model predictions also validates the mathematical solution described above. ${ }^{19}$

These two prior studies set the foundation for the measurement of full-tensor elastic constants from polycrystalline samples without the constraints of highly textured grains or completely randomly oriented grains. EBSD mapping will be employed to evaluate the crystallographic orientations perpendicular to the PDMS film grating for the few grains to be measured. The quite arbitrary orientations in polycrystalline samples, often away from high-symmetry ones, frequently lead to the simultaneous presence of several bulk and SAW modes; thus a highly reliable and efficient strategy needs to be developed for the computer algorithm to automatically identify the right SAW from all the wave modes. In addition, very efficient optimization procedures need to be implemented into the forward-calculation algorithm in order to most effectively optimize the input elastic constant values to obtain the best match to the measured SAW velocities along several crystallographic orientations.
This study intends to complete the development of the new technique to enable measurement of elastic constants from polycrystalline samples by integrating the above advances with EBSD orientation mapping and the development of efficient algorithms to handle arbitrary orientations as well as effective inverse optimization procedure/code. Direct comparison of the elastic constants obtained from polycrystalline samples of pure Al, $\mathrm{Co}, \mathrm{Fe}, \mathrm{Nb}, \mathrm{Ni}, \mathrm{Sn}$, and $\mathrm{Ta}$ with highly reliable values measured from single crystals will benchmark the accuracy for the new technique.

\section{Brief description of the models}

An algorithm based on elastodynamic equations was developed and coded in the MATLAB language to predict the SAW velocities along any arbitrary crystallographic orientation when the crystal structure, elastic constants, and density are given. ${ }^{19}$ Under the assumption of linear elastic deformation in a homogeneous anisotropic crystal in the absence of any body force, body torque, dissipative process or external fields, the governing equation is expressed as:

$C_{\mathrm{ijkl}} \frac{\partial^{2} U_{\mathrm{l}}}{\partial \mathrm{x}_{\mathrm{j}} \partial x_{\mathrm{k}}}=\rho \frac{\partial^{2} U_{\mathrm{l}}}{\partial t^{2}} \quad x_{3} \leq 0$

where $U$ is the displacement, $\rho$ crystal density, $C_{\mathrm{ijk}}$ the elastic constants, and $x$ and $t$ space and time, respectively. The $x_{3} \leq 0$ condition refers to a semi-infinite system and only the lower half of the crystal is considered. Here the Einstein summation convention is employed. When the restriction of half space is removed, the solutions to Eq. (1) correspond to the three (and only three) bulk wave modes: the L wave, the FT wave, and the ST wave, respectively. These bulk wave solutions from the unconstrained Eq. (1) are accomplished by expressing the displacement $U$ as an exponential function and then substituting it into Eq. (1) as elegantly summarized by Rosenbaum. ${ }^{21}$ Our bulk wave model is coded according to these solutions.

In order to solve for the surface waves, the initial and boundary conditions need to be set for the response of the surface displacement under a point stimuli exerted by an external force in a semi-infinite crystal. The evolution of the surface displacement provides information to predict the SAW velocities along any orientation of interest given the elastic constants, orientation, and density. The corresponding boundary conditions are set as:

$\left.\sigma_{\mathrm{i} 3}\right|_{z=0}=\left.C_{\mathrm{i} 3 \mathrm{k}} \frac{\partial U_{\mathrm{l}}}{\partial x_{\mathrm{k}}}\right|_{z=0}=\delta_{\mathrm{i} 3} \delta(x, y) \delta(t)$

$U_{\mathrm{i}}(x, y, z, t)=0, \quad$ when $x, y, z, t \rightarrow \infty$

where,

$\delta_{\mathrm{i} 3}=\left\{\begin{array}{cc}1 & i=3 \\ 0 & \mathrm{i} \neq 3\end{array}\right.$

$\delta(x, y)=\delta(x) \delta(y)$

and $i, j, k, l=1,2,3$

The corresponding initial condition is determined by the principle of causality

$$
U_{i}(x, y, z, t)=0 \text { for } t<0
$$

Boundary condition (2) refers to the beginning when the force only exerts on the origin and the stress only has a non-vanish component normal to the surface. Boundary condition (3) refers to the vanishing displacement on the surface at infinite time or space. Initial condition (7) refers to the zero displacement before any trigger (laser impingement).

Various ways to deal with such surface wave solutions are carefully reviewed by Every et al. ${ }^{22}$ and Favretto-Cristini et al. ${ }^{23}$ 
a

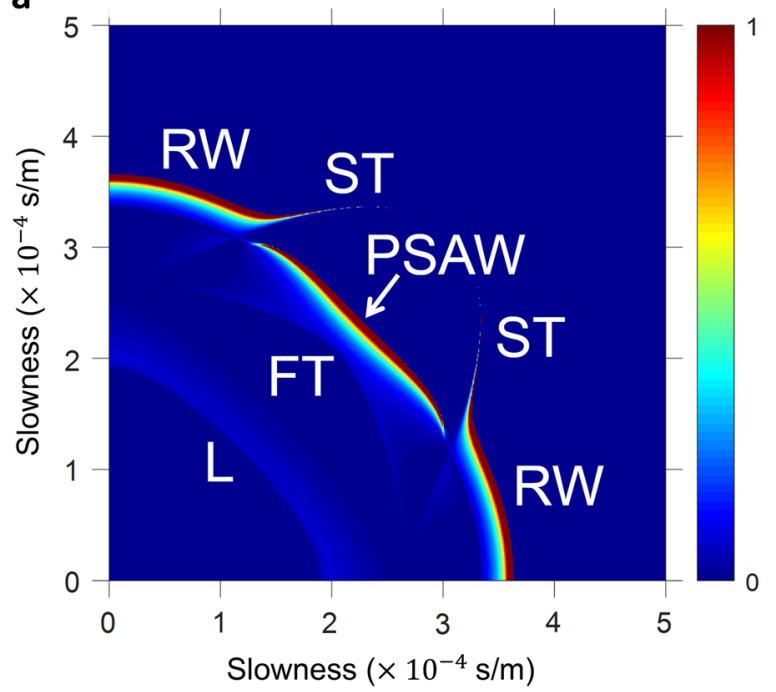

b

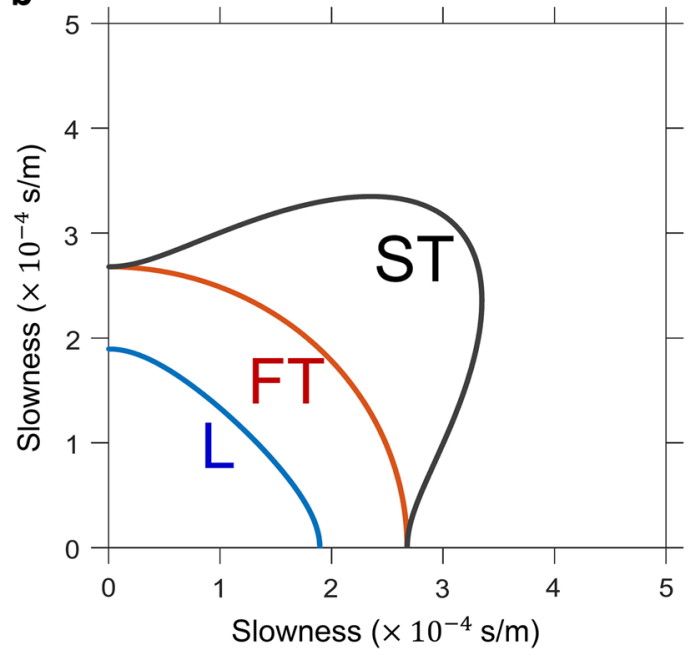

Fig. 1 Computed acoustic wave behavior on the (001) surface of pure Ni plotted in the slowness (reciprocal of velocity) space: a full solution with both surface waves and bulk waves; and $\mathbf{b}$ bulk wave solutions only. The fast-acting bulk wave solutions in $\mathbf{b}$ for the L, FT, and ST modes help the identification of the two surface waves: the RW and the PSAW. The color in a shows the relative magnitude of the wave displacement on the surface

What is adopted here is a method to perform the Fourier transform merely on the time and space parallel to the surface, leaving the third vertical component intact. ${ }^{19}$ Since only the wave velocities not the displacements are of interest, all the information can be obtained in the frequency domain after performing the Fourier transform. The inverse Fourier transform becomes unnecessary, saving a significant amount of computing time. Results from our surface modeling is consistent with that of Every et al. ${ }^{22}$ but our model is much easier to implement in computer coding, especially the ability to handle low-symmetry orientations that is essential for measurements on polycrystalline samples.

The capability of the models and algorithms in predicting all the bulk and SAW modes is demonstrated in Fig. 1, which shows results for pure $\mathrm{Ni}$ on its (001) plane and is plotted in the slowness space-the slowness is the reciprocal of the phase velocity by definition and it is widely used to simplify the plotting. A separate fast-acting model is used to compute the bulk waves (Fig. 1b) so they can be quickly identified from the general model result (Fig. 1a). By qualitatively matching those bulk wave modes in the surface modeling results, the rest two surface modes (RW and PSAW) can be identified quickly. A careful comparison of the bulkwave-only behaviors without the surface waves (Fig. 1b) with those in the presence of SAWs (Fig. 1a) shows that the FT wave behavior was slightly altered by the SAWs but the $L$ wave is unaffected; thus, the FT wave position matching between the bulk-wave-only results and those of full calculations can only be qualitative. The PSAW is "supersonic" since its velocity exceeds the limiting bulk velocity that is defined as the slowest bulk wave velocity, i.e., the velocity of the ST wave along the orientation of interest. In contrast, RW is "subsonic" and thus it can be identified along any given propagation orientation as the peak with a velocity lower (higher slowness) than that of the ST wave (only in extremely rare cases the RW velocity is "supersonic" and such situations can be identified by the full surface and bulk wave calculations). ${ }^{22,}{ }^{23}$ For orientations along which the RW is too weak to be observable, the PSAW is selected as the SAW. This is accomplished by a first search of the RW peak in the slowness region above that of the ST wave; and in the absence of an observable RW peak, the program/algorithm selects the first peak with the slowness lower than that of the ST wave. The SAW velocity is simply the reciprocal of the slowness of the identified SAW/PSAW peak.

The presence of multiple bulk and surface waves along a lowsymmetry orientation is shown in Fig. $2 \mathrm{~b}$ as an example, in contrast to a high-symmetry [010] orientation in Fig. 2a (the same orientation as the vertical axis of Fig. 1a), both for pure Ni. The slowness (reciprocal of velocity) of the ST wave is also computed for each orientation from the bulk wave model and is denoted as the dashed lines in Fig. 2. Even though the ST peak happens to be too weak to be observable in both orientations in Fig. 2, the computation of its position from the bulk wave model is essential to help identify the RW peak. The RW peak can be identified since it has lower velocity (higher slowness) than the corresponding ST wave along the same orientation.

Experimental measurements

EBSD was employed to map the orientation of the grains, which was performed with the XL-30 ESEM system (FEl, Inc.), which had a high-brightness field-emission electron gun. The EBSD detector and the associated imaging processing software were from TSL Inc. Microhardness indents were placed around a location of interest to enable the location and its orientation to be precisely referenced during EBSD as well as the subsequent TDTR measurement. The EBSD pattern of an $800 \times 1000 \mu \mathrm{m}$ region of the pure $\mathrm{Ni}$ sample taken with a step size of $8 \mu \mathrm{m}$ in both directions is shown in Fig. 3a, which provided the Euler angles at each pixel that connect the sample coordinate system with the crystallographic principal coordinate system. The rolling direction $(A 1,[100])$ is vertical and pointing upward; the transverse direction (A2, $[010])$ is horizontal and pointing left; and the normal direction [001] is vertically pointing out of the page/image, as shown in Fig. 3.

Assuming the PDMS film was placed with the parallel lines exactly vertically on Fig. $3 a$, then the SAW propagation direction would be exactly horizontal. The SAW velocity along this direction/ orientation for each pixel can be computed using the models and algorithms described in the previous section with the singlecrystal elastic constants and density of Ni from the literature as the input. Figure $3 \mathrm{~b}$ is the computed SAW velocity map that shows the expected SAW velocity values (some spots were not computed due to the poor quality of the EBSD indexing). During the actual experimental measurement, the PDMS film lines may be placed not exactly along the vertical direction (with the grating vector pointing horizontally), then the angle between the film grating vector and the horizontal line can be measured by referencing to the microhardness indents. It is then a straightforward 


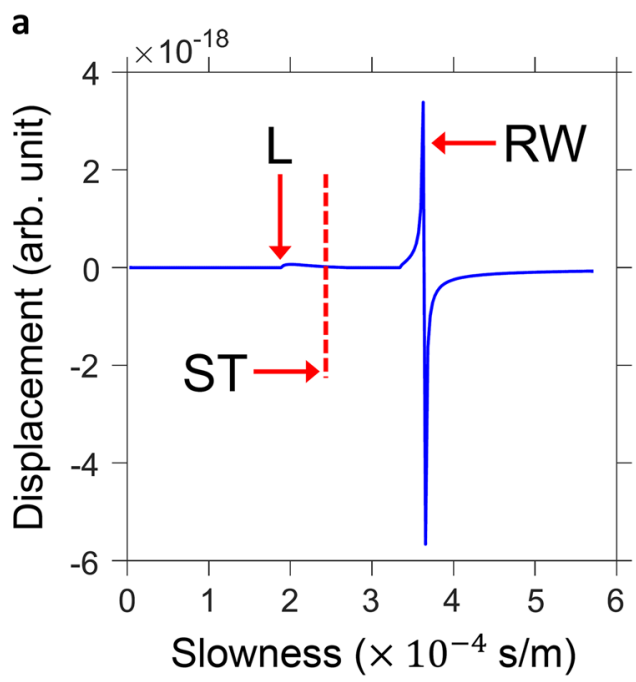

b

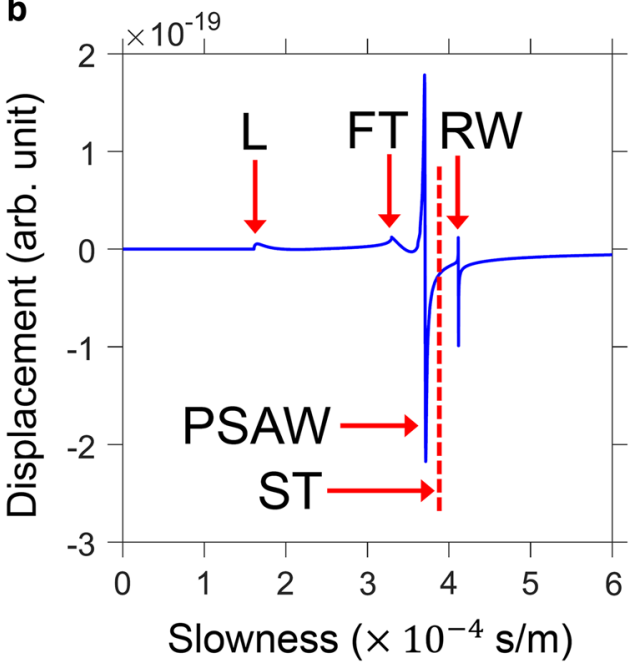

Fig. 2 Computed wave displacement as a function of slowness for pure $\mathrm{Ni}$ along a high-symmetry orientation [010] (a) and a lowsymmetry orientation $[0.69,0.59,-0.42](\mathbf{b})$, showing the presence of several wave modes along the low-symmetry orientation. The bulk wave modeling helps identify the bulk $\mathrm{L}$ and FT modes and can compute the slowness of the ST mode even though its displacement is too low to be observable along these two orientations. The computed ST mode information is essential for the identification of the SAW

mathematical rotation to obtain the real crystallographic orientations along which the SAW propagates in each grain. The real SAW propagation directions are essential input to the models to predict the SAW velocities.

After attaching the PDMS film on the polished surface of the samples where indents have been placed and the EBSD pattern has been taken, the TDTR measurements were then performed to obtain the SAW velocities from several grains. The TDTR measurement setup for the elastic constant measurement is the same type of system that has been employed for the measurements of several other thermophysical properties such as thermal conductivity, 24,25 specific heat capacity, ${ }^{26}$ and coefficient of thermal expansion. ${ }^{27}$ The TDTR laser system is described in detail elsewhere. ${ }^{19,26}$ A Ti-sapphire femtosecond laser, pumped by a semiconductor (Nd:YVO) laser, generated a laser beam whose wavelength was adjusted to be $780 \mathrm{~nm}$. The beam was split by a polarizing beam splitter into two beams with the pump beam a

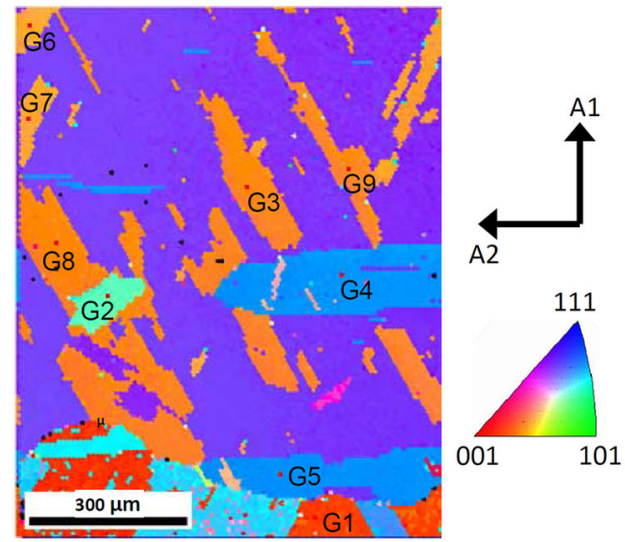

b

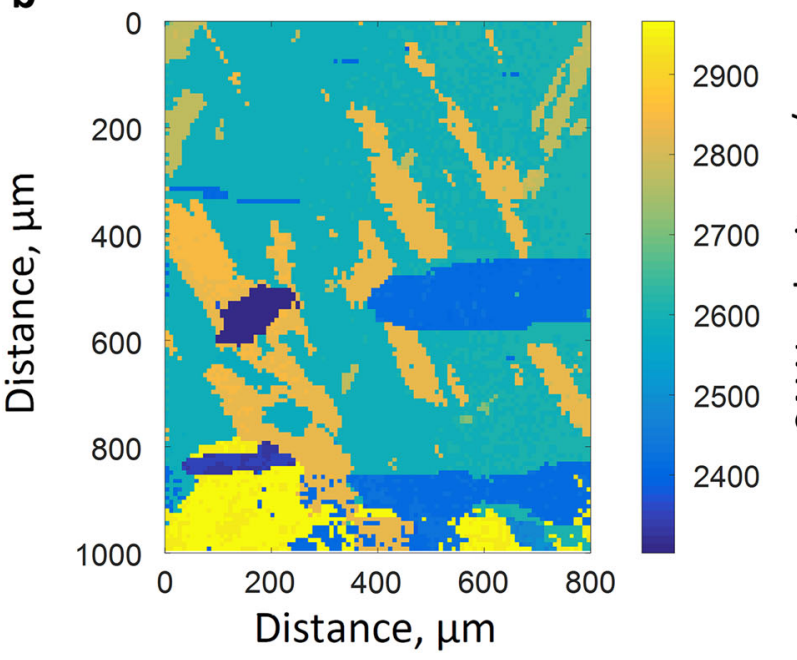

Fig. 3 EBSD orientation map (a) and the computed SAW velocity map (b) along horizontal propagation direction using the SAW solution model with the elastic constants and density of pure $\mathrm{Ni}$ as well as the EBSD orientation as the input to the model

being modulated to $9.3 \mathrm{MHz}$ using an electro-optic modulator and employed to trigger acoustic waves on the surface of a sample. The other beam, the probe beam, was used to detect the acoustic waves through the change of the surface displacement profile that was confined within the parallel grating lines of the PDMS film. The mechanism of the TDTR signal is explained in detail by $\mathrm{Li}$ et al. $^{20} \mathrm{~A}$ delay-stage was employed to adjust the optical path length travelled by the pump beam relative to the probe beam, and hence the time difference/delay of the arrivals of the two beams on the surface. The probe beam with a diameter of $\sim 5 \mu \mathrm{m}$ was placed at the exact location of the pump beam with about the same laser beam diameter. When the pump beam was moved away from the probe beam in this pump-probe measurement, the signal diminished very quickly, indicating that the generated SAW was confined close to the pump beam diameter and thus the spatial resolution of the measurement can be on the order of 10-15 $\mu \mathrm{m}$. A lock-in amplifier was used to assist the acquisition of both the in-phase and the out-of-phase signals for the wave velocity analysis. The entire process was controlled and monitored by a carefully programmed in-house LabVIEW VI (Virtual Instrument) code.

By adjusting the delay stage/time to vary the relative arrivals of the pump and the probe beams on the same spot on the sample surface, the TDTR in-phase signal intensity as a function of the delay time was obtained as shown in the example of Fig. 4a. The periodic modulation was due to the interaction of the SAW with 


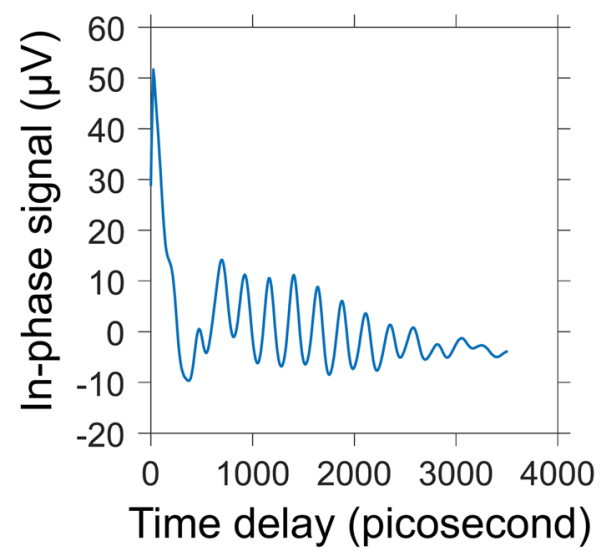

b

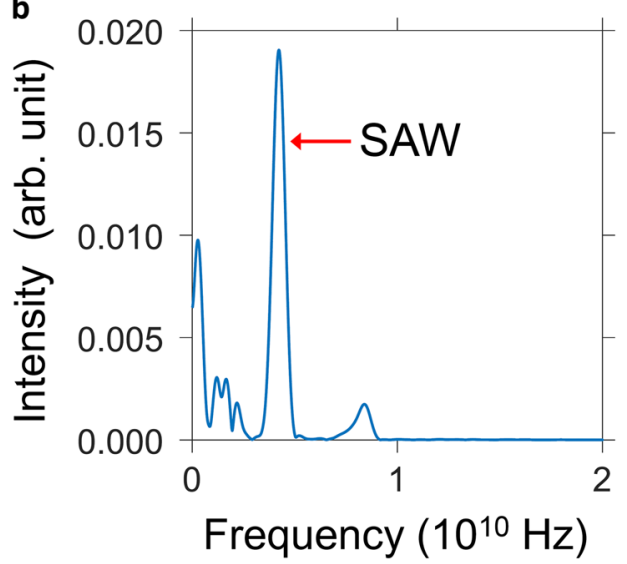

Fig. 4 Example outputs from a TDTR measurement showing in both the time domain (a) and the frequency domain (b). In the time domain, periodic modulation is due to the interaction of the SAW with the PDMS parallel line grating, which leads to a temporary standing wave before it dies out. The SAW velocity is equal to the wavelength $(700 \mathrm{~nm})$ times the SAW frequency

the PDMS grating structure. The intensity was gradually dying out because the heat was flow inward to the sample. A Fourier transform of the signal in Fig. $4 a$ yielded a few peaks in the frequency domain as shown in Fig. 4b. The highest peak corresponds to the SAW mode. The higher-frequency lowerintensity peak, if it emerged, was the result of the harmonics of the PDMS film since it was always at exactly twice the frequency of the SAW peak. A few lower frequency peaks were always present and some were associated with the eigenmodes of the PDMS film itself, and could be easily identified since their positions did not change from one sample to another. Since the acoustic wavelength is equal to the period of the PDMS grating film $\left(700 \mathrm{~nm}\right.$ in this case), the experimental SAW velocity $\left(V_{\text {expt }}\right)$ is simply the product of wavelength $(700 \mathrm{~nm})$ times the frequency of the SAW.

Since the SAW velocities were measured under the physical sample coordinate system while the models predict the velocities under the crystallographic principal coordinate system, it is necessary to convert the EBSD Euler angles through three consecutive rotations to bring the sample coordinate system to be coincidence with the principal coordinate system of the crystals.

\section{RESULTS}

Based on the EBSD orientation map and the indents placed on the pure $\mathrm{Ni}$ sample, nine grains were selected for the TDTR
Table 1. Comparison of the SAW velocities between experiments and modeling

\begin{tabular}{lllll}
\hline Grain & Location $(x, y), \mu \mathrm{m}$ & $V_{\text {model, }} \mathrm{m} / \mathrm{s}$ & $V_{\text {expt }} \mathrm{m} / \mathrm{s}$ & Difference (\%) \\
\hline G1 & 560,950 & 2976.2 & 2886.7 & 3.1 \\
G2 & 170,520 & 2437.3 & 2396.0 & 1.7 \\
G3 & 435,340 & 2902.2 & 2914.3 & -0.4 \\
G4 & 589,500 & 2621.7 & 2612.7 & 0.3 \\
G5 & 738,889 & 2629.6 & 2640.3 & -0.4 \\
G6 & 22,32 & 2868.9 & 2812.3 & 2.0 \\
G7 & 25,209 & 2866.5 & 2808.1 & 2.1 \\
G8 & 78,446 & 2907.0 & 2897.3 & 0.3 \\
G9 & 630,306 & 2895.0 & 2899.4 & -0.2 \\
\hline Note: The location is the $x$ - $y$ coordinates (in $\mu \mathrm{m}$ ) relative to the origin which \\
is the upper left corner of Fig. 3
\end{tabular}

measurements as marked on Fig. 3a with the measured SAW velocities $\left(V_{\text {expt }}\right)$ listed in Table 1 . During these measurements, large grains were intentionally selected for the measurement since it is easier to locate them in the TDTR system using the indents as references. Reliable SAW velocity measurements can be made in smaller (e.g., $30 \mu \mathrm{m}$ diameter) grains as long as they can be located accurately during the TDTR measurements to allow accurate placement of the laser beams in the center of the grains to avoid any boundary effect. Since the actual crystallographic orientations along which the SAW propagated can be obtained from the EBSD data, the SAW velocities for each grain can be computed using the literature elastic constants of pure $\mathrm{Ni}^{28}$ as shown in Table 1 as $V_{\text {model }}$. The $V_{\text {model }}$ and $V_{\text {expt }}$ are within $5 \%$ for all the nine grains, indicating that both models and the measurements are very reliable.

In order to obtain the elastic constant values from the measured SAW velocities $\left(V_{\text {expt }}\right)$ of the nine grains with known crystallographic orientations from EBSD (without using the $C_{\mathrm{ij}}$ values of pure $\mathrm{Ni}$ from the literature), an efficient optimization algorithm must be developed to input various starting values of elastic constants to the models to compute the SAW velocities $\left(V_{\text {model }}\right)$ in order to achieve the best match with the experimental measurements along the nine orientations.

For a cubic crystal, there are only three independent elastic constants $C_{\mathrm{ij}}\left(C_{11}, C_{12}\right.$, and $\left.C_{44}\right)$ to be determined and thus the regular optimization process worked well: an initial set of $C_{\mathrm{ij}}$ values were randomly selected by the computer and input into the models to predict the SAW velocities along the nine orientations. The summation of the difference between $V_{\text {model }}$ and $V_{\text {expt }}$ over all the measured orientations $\Delta=\Sigma_{i}\left|\left(V_{\text {model }}-V_{\text {expt }}\right) / V_{\text {expt }}\right|$ was computed. Then the algorithm kept updating the $C_{\mathrm{ij}}$ values until the $\Delta$ reached a minimum, which represents a local optimum set of elastic constants. Since the algorithm might have reached only a local optimum, multiple initial sets of $C_{i j}$ values were tested to reach a more "global" optimum and the resultant $C_{\mathrm{ij}}$ values were taken as the final experimentally measured elastic constants. The results for pure $\mathrm{Ni}$ are shown in the first row in Table 2. The difference between our $C_{11}, C_{12}$, and $C_{44}$ values measured from the polycrystalline sample shown in Fig. 3 with these reported in the literature that were measured from single crystals ${ }^{28}$ is only 3.4, $-2.2,-0.2 \%$, respectively.

In addition to $\mathrm{Ni}$ (measured on nine grains with the minimum grain dimension of $\sim 60 \mu \mathrm{m})$, similar measurements have been performed on pure Al (nine grains with the minimum grain dimension of $\sim 100 \mu \mathrm{m}$ ), Ta (six grains with the minimum grain dimension of $\sim 40 \mu \mathrm{m}$ ), $\mathrm{Nb}$ (six grains with the minimum grain dimension of $\sim 240 \mu \mathrm{m}$ ), and bcc Fe (eight grains with the minimum grain dimension of $\sim 100 \mu \mathrm{m}$ ) as representative cubic 


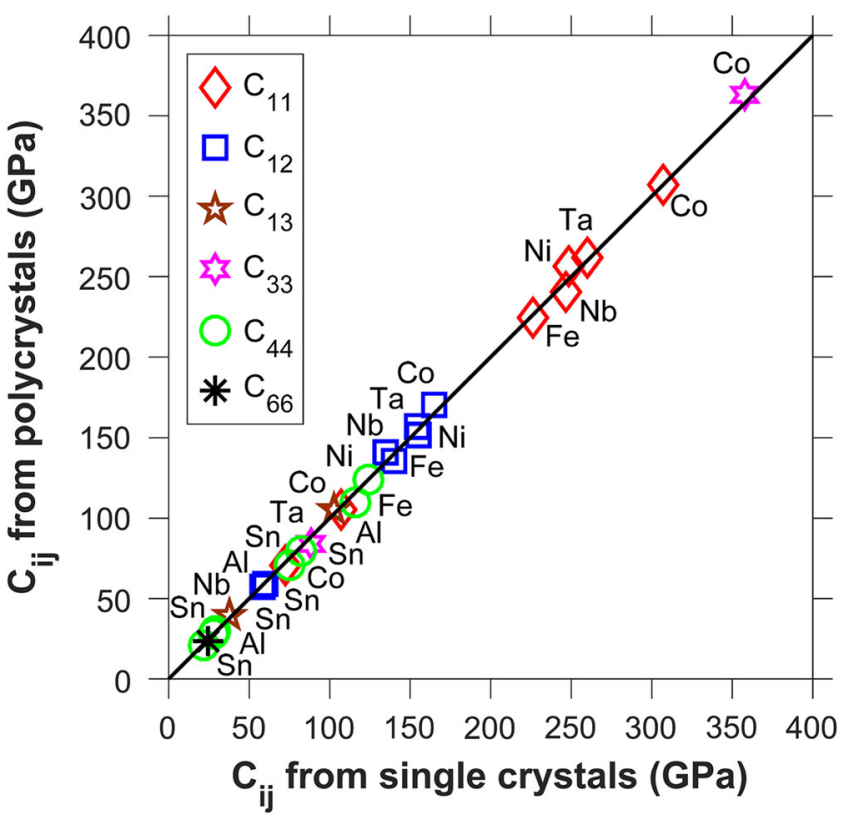

Fig. 5 Comparison of the elastic constants measured from polycrystalline $\mathrm{Al}, \mathrm{Co}, \mathrm{Fe}, \mathrm{Nb}, \mathrm{Ni}, \mathrm{Sn}$, and $\mathrm{Ta}$ samples using ultrafast laser-generated SAWs with those reported in the literature measured from single crystals. The single crystal data are from refs. 28-34

materials. The SAW signal is very weak for Ta, so a very thin Al film (less than $20 \mathrm{~nm}$ ) was deposited as an effective transducer to enhance the signal intensity. The elastic constants of $\mathrm{Al}$ have a very low anisotropy, our normal starting value setting for the optimization can only extract $C_{44}$ and the anisotropic ratio $2 C_{44} /\left(C_{11}-C_{12}\right)$ accurately. More accurate $C_{11}$ and $C_{12}$ were obtained through decreasing step size and increasing the number of the initial values.

The measured $C_{\mathrm{ij}}$ values from all five cubic polycrystalline pure metals are summarized in Table 2 in comparison with results from single crystal measurements, ${ }^{28-32}$ showing that the maximum difference is only $5.3 \%$ among all the 15 measured $C_{\mathrm{ij}}$ values with the standard deviation of difference between the polycrystalline (this study) and single-crystal (the literature) measurements for $C_{11}, C_{12}$, and $C_{44}$ being $2.2,3.2,3.5 \%$, respectively. The excellent agreement is graphically shown in Fig. 5 .

For non-cubic materials, we prepared and measured a hexagonal pure Co sample (measured on 10 grains with 3 PDMS orientations with the minimum grain dimension of $\sim 30 \mu \mathrm{m}$ ) and tetragonal $\beta-S n$ sample (measured on 11 grains with 3 PDMS orientations with the minimum grain dimension of $\sim 30 \mu \mathrm{m})$. The PDMS film rotations enabled us to measure SAW velocities along more distinct crystalographic orientations in order to obtain elastic constants with more distinct components $\left(C_{11}, C_{12}, C_{13}, C_{33}\right.$, and $C_{44}$ for $C_{0}$; and $C_{11}, C_{12}, C_{13}, C_{33}, C_{44}$, and $C_{66}$ for $\beta-S n$ ).

The pure Co sample was heat treated at $900^{\circ} \mathrm{C}$ for $100 \mathrm{~h}$ and then furnace cooled to ambient temperature to form the hcp Co phase. The TDTR signal was generally small but observable regardless of the orientations of the SAW measurements. The signal for some grain-PDMS combinations was noisy; thus, only 16 effective measurements were obtained for the elastic constant evaluation. The elastic constants obtained from this study are compared in Table 2 with those obtained by McSkimin ${ }^{33}$ from single crystals. The results clearly show the largest difference is only $6.8 \%$ for $C_{44}$, and all other values are within $3 \%$.

The pure Sn sample was heat treated at $200^{\circ} \mathrm{C}$ for $100 \mathrm{~h}$ and then furnace cooled to ambient temperature to make sure the tetragonal $\beta-S n$ phase was obtained and the grain size was large 
enough for measurements. The TDTR signal is quite strong and the measurements comprised 33 grain-PDMS film orientation combinations. The elastic constants obtained from this study are listed in Table 2 in comparison with the results reported by Kammer et al., ${ }^{34}$ showing an excellent agreement with the largest deviation of only $5.6 \%$ for $C_{13}$, which is reasonable since $C_{13}$ is the least sensitive one. ${ }^{34}$

\section{DISCUSSION}

In order to save computation time, especially considering that the number of independent elastic constants increases with decreasing crystal symmetry, a neural network machine learning program was developed to accelerate the optimization process and to increase the robustness of the final elastic constant values. An examination of the compilations of experimental elastic constants of cubic crystals reveals that the ranges of $C_{11}, C_{12}$, and $C_{44}$ are about $4-800 \mathrm{GPa}, 2-300 \mathrm{GPa}$, and $2-300 \mathrm{GPa}$, respectively. By systematically inputting the $C_{i j}$ values in these ranges and compute the corresponding $V_{\text {model, }}$ a big data set was generated of elastic constants and SAW velocities $\left(V_{\text {model }}\right)$. This data set was employed to train the network to find the order inside it. The network training was performed through the MATLAB neural network tool box. The neural network algorithm allowed us to just input the experimental velocities into the network and get back the elastic constants immediately. The neural network results agree with our forward-calculation results.

The size of our data set used to train the neural network depends on crystalline symmetry. For cubic materials such as $\mathrm{Ni}$, about 6000 elastic constant-SAW velocity pairs were generated for each orientation with the elastic constants varying from 4-800 GPa for $C_{11}, 2-300 \mathrm{GPa}$ for $C_{12}$, and 2-300 GPa for $C_{44}$, respectively. The Born stability criteria ${ }^{35}$ were guaranteed during the random generation of $C_{\mathrm{ij}}: C_{11}-\left|C_{12}\right|>0 ; C_{11}+2 C_{12}>0$; and $C_{44}>0$.

Our network architecture employs one hidden layer and one output layer. The input data presented to the network are those SAW velocities generated through our SAW model and the elastic constants, which work as the target data (desired network output), used to produce those velocities. They form the elastic constantvelocity pairs provided to train the network. Of the total generated data, $70 \%$ goes to the training set, $15 \%$ goes to the validation set, and the remaining $15 \%$ goes to the testing set. The objective function is to minimize the difference between the desired elastic constants provided by us and the returned elastic constants trained from the neural network. The training algorithm used here is Levenberg-Marquardt. The training process completes once the mean square error of the validation set starts to increase.

To make sure the model is not over fitted, different numbers of neurons were tested in the hidden layer. When the training result has a very high $R$ value while the testing result has a very low $R$ value, it is over fitted, and thus the number of neurons used in the hidden layer is reduced accordingly. In contrast, when the training result has a very low $R$ value, it is under fitted, and thus the number of neurons is increased accordingly. Such trial-and-error fitting leads to an optimal number of $\sim 26$ neurons in the hidden layer to have an $R$ value above 0.95 for all the training set, validation set, and testing set.

\section{CONCLUSIONS}

An easy-to-use technique is developed to measure single-crystal elastic constants from a polished surface of polycrystalline samples without the need of growing single crystals which is usually very time-consuming and labor intensive. The new technique is made possible by integrating: (1) a robust mathematical solution for SAWs and computer code to compute the SAW velocity along any crystallographic orientation of any crystal structure with given elastic constants and density; (2) an easy-to-use PDMS film grating that suppresses the dispersion due to the broadband signal and isolates one SAW parallel to the PDMS film grating vector direction to be measurable; (3) accurate measurement of the SAW frequency from the TDTR signals; (4) accurate measurement of crystallographic orientations using EBSD; and (5) a robust algorithm/code to optimize the elastic constants from the orientation-dependent SAW velocities. Measurements on polycrystalline samples of 7 pure metals show that the 26 elastic constants measured during this study are all within $6.8 \%$ of the single-crystal measurements reported in the literature. This SAW-PDMS-TDTR-MODEL technique has a spatial resolution about $10-15 \mu \mathrm{m}$ and has demonstrated reliable measurements from $30 \mu \mathrm{m}$ diameter/width grains. The methodology is applicable to any crystal structures and can contribute significantly to the establishment of elastic constant databases for the Materials Genome Initiative ${ }^{36}$ and for the accelerated materials design using the Integrated Computational Materials Engineering approach. ${ }^{37}$

\section{MATERIALS AND METHODS}

The polycrystalline pure metals employed for this study were all purchased from commercial sources: Al (99.9995 wt.\% purity) from Alfa Aesar; Co (99.95 wt.\% purity), Fe (99.95 wt.\% purity), Sn (99.95 wt.\% purity), and $\mathrm{Ni}$ ( 99.95 wt.\% purity) from the Micron Metals, Inc.; and Nb ( 99.95 wt.\% purity) and Ta (99.95 wt.\%) from Kamis Inc. High-quality metallographic surface finish is required for both EBSD orientation imaging and the TDTR measurement. The samples all went through the usual metallographic preparation procedures including grinding with progressively finer grit $\mathrm{SiC}$ grinding papers (up to 1200 grit) and polishing with $1 \mu \mathrm{m}$ diamond paste. A final step was the usual process for EBSD sample preparation, either a vibratory polishing with $0.05 \mu \mathrm{m}$ colloidal silica spheres suspended in water or an electro-polishing for very soft metals such as Al.

The PDMS films of about $1 \times 1 \mathrm{~cm}$ squares were prepared from a pure $\mathrm{Si}$ grating mold with parallel groves of $700 \mathrm{~nm}$ periodicity and $50 \%$ duty cycle (SNS-C14.3-0808-350-D45-P, LightSmyth Technology). The PDMS solution (Sylgard 184, Dow Corning) and its curing agent with a ratio 10:1 were well-mixed and poured onto the Si wafer mold, degassed at ambient temperature in a desiccator linked to a mechanical vacuum pump, and then cured at $80^{\circ} \mathrm{C}$ for at least 1 day. The PDMS film was then peeled off from the Si mold and placed onto the polished surface of the samples with the PDMS film grating lines touching the surface. The PDMS film worked like a set of comb transducers, playing the role of confining the acoustic mode propagating along the direction parallel to the film grating vector with a wavelength the same as the film periodicity. The PDMS film fabrication process is mostly following the literature practice, but modified with one formula instead of two. ${ }^{20}$

\section{ACKNOWLEDGEMENTS}

The authors are thankful to Dr. Peng Zhao for his help in the SAW modeling, Dr. Changdong Wei for his help in optimization of the TDTR experimental setup, Dr. David Cahill and Dr. Dongyao Li for very valuable discussions, and Dr. Jie Kuang for his help in mathematical optimization procedures. The models and algorithms are implementation in MATLAB codes, which are available upon request from the authors. This study was funded by U.S. National Science Foundation under grant number NSF-DMR-1237577.

\section{AUTHOR CONTRIBUTIONS}

X.D. performed all the experimental work and computational modeling as part of his Ph.D. dissertation research. J.C.Z. came up with the overall idea and advised the study. Both contributed substantially to the manuscript preparation.

\section{COMPETING INTERESTS}

The authors declare no competing interests.

\section{REFERENCES}

1. Mura, T. Micromechanics of Defects in Solids, 2nd edn (Kluwer Academic/Springer, 1987).

2. Schuren, J. C. et al. New opportunities for quantitative tracking of polycrystal responses in three dimensions. Curr. Opin. Solid State Mater. Sci. 19, 235-244 (2015). 
3. Chen, L.-Q. Phase-field models for microstructure evolution. Annu. Rev. Mater. Sci. 32, 113-140 (2002).

4. Wang, Y. \& Li, J. Phase field modeling of defects and deformation. Acta. Mater. 58, 1212-1235 (2010).

5. De Jong, M. et al. Charting the complete elastic properties of inorganic crystalline compounds. Sci. Data 2, 150009 (2015).

6. Liu, G. \& Maynard, J. D. Measuring elastic constants of arbitrarily shaped samples using resonant ultrasound spectroscopy. J. Acoust. Soc. Am. 131, 2068-2078 (2012).

7. Migliori, A. \& Sarrao, J. L. Resonant Ultrasound Spectroscopy (Wiley, 1997).

8. Benedek, G. B. \& Fritsch, K. Brillouin scattering in cubic crystals. Phys. Rev. 149, 647-662 (1966).

9. Yamaguchi, M. et al. Brillouin scattering study of gallium nitride : elastic stiffness constants. J. Phys. Conden. Matter 9, 241-248 (1997).

10. White, P. S., Rodgers, J. A. \& Le Page, Y. CRYSTMET: a database of the structures and powder patterns of metals and intermetallics. Acta Crystall. B B58, 343-348 (2002).

11. Xu, Y., Aizawa, T. \& Kihara, J. Structure of surface wave on coarse-grained nickel observed by acoustic spectro-microscopy. Mater. Trans. JIM 37, 1690-1698 (1996).

12. Xu, Y., Aizawa, T. \& Kihara, J. Simultaneous determination of elastic constants and crystallographic orientation in coarse-grained nickel by acoustic spectromicroscopy. Mater. Trans. JIM 38, 536-545 (1997).

13. Gasteau, D. et al. Single crystal elastic constants evaluated with surface acoustic waves generated and detected by lasers within polycrystalline steel samples. J. Appl. Phys. 119, 043103 (2016).

14. Li, D. Y. \& Szpunar, J. A. Determination of single crystals' elastic constants from the measurement of ultrasonic velocity in the polycrystalline material. Acta Metall. Mater. 40, 3277-3283 (1992).

15. Tane, M., Yamori, K., Sekino, T. \& Mayama, T. Impact of grain shape on the micromechanics-based extraction of single-crystalline elastic constants from polycrystalline samples with crystallographic texture. Acta. Mater. 122, 236-251 (2017).

16. Pathak, S. \& Kalidindi, S. R. Spherical nanoindentation stress-strain curves. Mater. Sci. Eng. R 91, 1-36 (2015).

17. Phani, M. K., Kumar, A., Jayakumar, T., Arnold, W. \& Samwer, K. Mapping of elasticity and damping in an $\alpha+\beta$ titanium alloy through atomic force acoustic microscopy. Beilstein J. Nanotechnol. 6, 767-776 (2015).

18. Morales-Rivas, L. et al. Nanomechanical characterization of nanostructured bainitic steel: peak force microscopy and nanoindentation with AFM. Sci. Rep. 5, 17164 (2015).

19. Zhao, P., Zhao, J.-C. \& Weaver, R. Dynamic surface acoustic response to a thermal expansion source on an anisotropic half space. J. Acoust. Soc. Am. 133, 2634-2640 (2013).

20. Li, D., Zhao, P., Zhao., J.-C. \& Cahill, D. G. Generation and detection of gigahertz surface acoustic waves using an elastomeric phase-shift mask. J. Appl. Phys. 114, 143102 (2013).

21. Rosenbaum, J. F. Bulk Acoustic Wave Theory and Devices (Artech House, 1988).

22. Every, A. G., Kim, K. Y. \& Maznev, A. A. The elastodynamic response of a semiinfinite anisotropic solid to sudden surface loading. J. Acoust. Soc. Am. 102, 1346-1355 (1997).
23. Favretto-Cristini, N., Komatitsch, D., Carcione, J. M. \& Cavallini, F. Elastic surface waves in crystals. Part 1: review of the physics. Ultrasonics 51, 653-660 (2011).

24. Huxtable, S., Cahill, D. G., Fauconnier, V., White, J. O. \& Zhao, J.-C. Thermal conductivity imaging at micrometre-scale resolution for combinatorial studies of materials. Nat. Mater. 3, 298-301 (2004).

25. Zhao, J.-C., Zheng, X. \& Cahill, D. G. Thermal conductivity mapping of the Ni-Al system and the beta-NiAl phase in the Ni-Al-Cr system. Scr. Mater. 66, 935-938 (2012).

26. Wei, C., Zheng, X., Cahill, D. G. \& Zhao, J.-C. Invited article: micron resolution spatially resolved measurement of heat capacity using dual-frequency timedomain thermoreflectance. Rev. Sci. Instr. 84, 071301 (2013).

27. Zheng, X., Cahill, D. G., Weaver, R. \& Zhao, J.-C. Micron-scale measurements of the coefficient of thermal expansion by time-domain probe beam deflection. J. Appl. Phys. 104, 073509 (2008).

28. Epstein, S. G. \& Carlson, O. N. The elastic constants of nickel-copper alloy single crystals. Acta Metall. 13, 487-491 (1965).

29. Thomas, J. F. Third-order elastic constants of aluminum. Phys. Rev. 175, 955-962 (1968).

30. Soga, N. Comparison of measured and predicted bulk moduli of tantalum and tungsten at high temperatures. J. Appl. Phys. 37, 3416-3420 (1966).

31. Bolef, D. I. Elastic constants of single crystals of the bcc transition elements $\mathrm{V}, \mathrm{Nb}$, and Ta. J. Appl. Phys. 32, 100-105 (1961).

32. Leese, J. \& Lord, A. E. Jr. Elastic stiffness coefficients of single-crystal iron from room temperature to $500^{\circ} \mathrm{C}$. J. Appl. Phys. 39, 3986-3988 (1968).

33. McSkimin, H. J. Measurement of the elastic constants of single crystal cobalt. J. Appl. Phys. 26, 406-409 (1955).

34. Kammer, E. W., Cardinal, L. C., Vold, C. L. \& Glicksman, M. E. The elastic constants for single-crystal bismuth and tin from room temperature to the melting point. J. Phys. Chem. Solids 33, 1891-1898 (1972).

35. Grimvall, G., Magyari-Köpe, B., Ozolinš, V. \& Persson, K. A. Lattice instabilities in metallic elements. Rev. Mod. Phys. 84, 945-986 (2012).

36. National Science and Technology Council. Materials genome initiative for global competitiveness. Office of Science and Technology Policy (Washington, DC, 2011).

37. Allison, J., Backman, D. \& Christodoulou, L. Integrated computational materials engineering: a new paradigm for the global materials profession. JOM 58, 25-27 (2006).

Open Access This article is licensed under a Creative Commons Attribution 4.0 International License, which permits use, sharing, adaptation, distribution and reproduction in any medium or format, as long as you give appropriate credit to the original author(s) and the source, provide a link to the Creative Commons license, and indicate if changes were made. The images or other third party material in this article are included in the article's Creative Commons license, unless indicated otherwise in a credit line to the material. If material is not included in the article's Creative Commons license and your intended use is not permitted by statutory regulation or exceeds the permitted use, you will need to obtain permission directly from the copyright holder. To view a copy of this license, visit http://creativecommons. org/licenses/by/4.0/.

(c) The Author(s) 2017 